\title{
Sense of time and interpretation of the evolution of time
}

\author{
Dhananjay Pal \\ Ex-Dean of research, Pharmacy College, Bengal School of Technology, Chuchura, Dist.-Hooghly, West Bengal, INDIA
}

Email address:

dhananjay.pal123@gmail.com, paldhananjay46@yahoo.com

\section{To cite this article:}

Dhananjay Pal. Sense of Time and Interpretation of the Evolution of Time. American Journal of Modern Physics. Vol. 2, No. 6, 2013, pp. 314-321. doi: 10.11648/j.ajmp.20130206.17

\begin{abstract}
Our consciousness model involving thought-carrying particle (TCP), thought retaining particle (TRP) and thought force $\left(\mathrm{T}_{\mathrm{F}}\right)$ signifies the existence of universal consciousness that exists along with the universe. This universal consciousness is a functional state of the universal mind. This universal mind (UM) is evolved at the Big Bang from void. The UM is constituted by these TCP and TRP in the inherent presence of thought force $\left(T_{F}\right)$. Thought force $\left(T_{F}\right)$ is an expression of universal consciousness. The Thought force $\left(\mathrm{T}_{\mathrm{F}}\right)$ being the primordial quantum field functions as the original super-force. $\mathrm{T}_{\mathrm{F}}$ being the original super-force functions as the origin of all the fundamental fields. TCP is the carrier of thought force $\left(\mathrm{T}_{\mathrm{F}}\right)$ that, in turn, appears to be the origin of all the fields. The quantized energy $\left(\varepsilon_{T}\right)$ of TCP is responsible to cause the universal consciousness as well as the cosmic microwave background radiation temperature. The individual consciousness owes its origin to the universal consciousness created by the same $\varepsilon_{T}$. The same $\varepsilon_{T}$ is the energy responsible for generating thought force $\left(\mathrm{T}_{\mathrm{F}}\right) . \mathrm{T}_{\mathrm{F}}$ being an expression of the universal consciousness is applicable to any inanimate object as well as to any biological system (having thinking ability). The $\mathrm{T}_{\mathrm{F}}$ exerts its functions both in vitro and in vivo. Time of any kind cannot exist without the existence of consciousness. Time without consciousness is not possible. But consciousness without time is possible. The manifestation of any type of time is solely dependent on the manifestation of consciousness. There is no manifestation of 'time' unless there is the generation of consciousness $\left[=\varepsilon_{T}=m_{T} c^{2}=h c / \lambda_{T}\right.$ (where $m_{T}=$ quantized mass of the TCP, $\mathrm{c}=$ free- space velocity of light, $\mathrm{h}=$ Planck's quantum constant, $\lambda_{T}=$ wavelength of the TCP)]. The origin of the physical time appears to be the origin of evolution of light and its evolved velocity (c) at that point of the beginning of universe. [This $\mathrm{c}$ is related with consciousness $\left(=\mathcal{E}_{T}\right)$ as shown here: $\left.\mathcal{E}_{T}=m_{T} c^{2}=h c / \lambda_{T}\right]$.
\end{abstract}

Keywords: Cosmic Microwave Background Radiation (CMBR), Universal Mind (UM), Thought Force (TF), Though-Carrying Particle (TCP), Thought Retaining Particle (TRP), Quantized Energy $\left(\varepsilon_{T}\right)$ of TCP

\section{Introduction}

It is most relevant and important to indicate the names of various eminent physicists like Erwin Schrödinger, Eugene Wigner, Brian Josephson, John Wheeler, Roger Penrose, Henry P Stapp, Freeman J. Dyson, Paul Davies, David Bohm, Basil Hiley, Fritjof Capra, Fred Alan Wolf and Amit Goswami who have addressed the inclusion of consciousness in their work. Consciousness is to be taken into account.

Consciousness model of Pal et al [1-3] involving TCP, TRP and thought force $\left(\mathrm{T}_{\mathrm{F}}\right)$ signifies the existence of universal consciousness that exists along with the universe.

Bhaumik [4] mentioned, "It would be reasonable to presume that the universe originated in a unity of all fields at or near Planck's dimension. John Wheeler strongly believes that "in defining any useful concept of reality" we have to take into account "the indispensable place of the participating observer---evidenced in quantum mechanics," the foregoing cannot be really true unless consciousness is as essential as aspect of nature as are the fields that give rise to force and matter and the primary field that gave rise to them".

Physicists determined that underlying quantum fields give birth to elementary particles. Bhaumik [4] mentioned that Frank Wilczek pointed out, "In quantum field theory, the primary elements of reality are not individual particles, but underlying fields. Thus, for example, all electrons are but excitations of an underlying field, naturally called electric field". The same holds true for all the fundamental particles of which matter is made.

TCP cannot exist without TRP and vice versa. Many physicists believe that unifying all the forces, including 
gravity, into a single theory would require a phenomenon called super-symmetry. With super-symmetry, every fermion would have a boson twin, and vice-versa. TCP that behaves like boson should accompany its super-symmetrical partner TRP that functions like fermion in the generalized simpler way. Thus TCP like boson cannot have anti-particle. But TRP that functions like fermion should have its anti-particle and here it is shown as Anti-TRP (see Scheme-I). It is to be noted that these TCP and TRP function like wavicle: wave-particle duality.

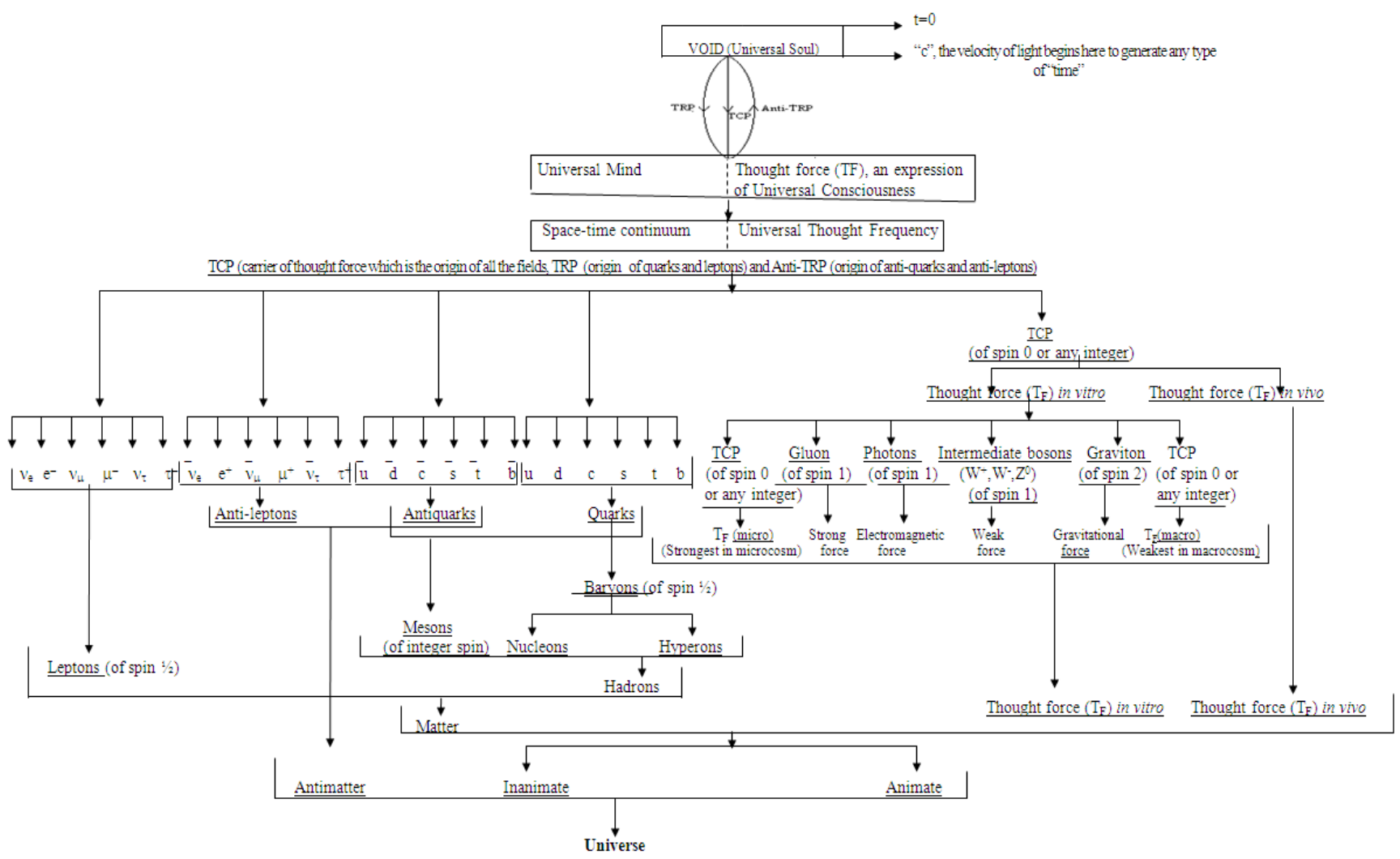

Scheme-I: Schematic presentation showing the evolution of the universe from the Void incorporating the postulated TCP (Thought-carrying particle), TRP (Thought-retaining particle) and Thought Force $\left(T_{F}\right)$. Laws of absolute conservation of mass, charge (and color) are to be maintained in order to develop a general theory for the unification of physics which would be freely applicable to the more general situations involving both the non-living system and living system having consciousness. Here TCP is the carrier of thought force $\left(T_{F}\right)$ that is the origin of all the fields. TRP is the origin of all the matter particles. It is to be noted that these TCP and TRP function like wavicle: wave-particle duality. Here thought force ( $\left.T_{F}\right)$ is an expression of the universal consciousness.

1.1. In contrast to the usual linear sequence of matter, body, life, brain, mind, consciousness, here the proposed cyclic sequence is first universal consciousness (a functional state of the universal mind), and then matter, body, life, brain, and regeneration of mind and consciousness. The evolution of life with mind and consciousness is possible purely due to the inherent existence of universal consciousness which exists along with the universe. The human nervous system is evolved to provide an appropriate material structure to individualize the universal consciousness, a characteristic of reality, pervading all manifestations.

Consciousness model of Pal et al [1-3] involving TCP, TRP and thought force $\left(\mathrm{T}_{\mathrm{F}}\right)$ signifies the existence of universal consciousness that exists along with the universe. Pal et al [3] showed that the functional state of Universal Mind (UM) is the universal consciousness that exists along with the universe. Pal et al [3] explained that the UM is evolved at the Big Bang from the eternal Void (see Scheme-I). This Void, in turn, is the source of infinite energy. And this UM is a finer matter. The individual mind being a constituent of the
$\mathrm{UM}$ is also a finer matter. The constituents of the UM and individual mind are the same. The ultimate constituents of matter and mind are the same as both mind and matter are aspects of one fundamental reality, which is called UM. The brain is the mediating link or interface between the individual mind and body.

Pal et al [3] explained that the constituents of the UM are the ultimate constituents of matter itself as everything in this universe is a manifestation of this UM. Pal et al [3] expressed that the UM is constituted by these TCP and TRP in the inherent presence of thought force $\left(\mathrm{T}_{\mathrm{F}}\right)$. Pal et al $[1,3]$ further explained that the ultimate constituents of matter and mind are these TCP and TRP in the inherent presence of thought force $\left(\mathrm{T}_{\mathrm{F}}\right)$ in vitro and thought force $\left(\mathrm{T}_{\mathrm{F}}\right)$ in vivo [See Scheme-I]. Pal [5] explained the existence of the thought force $\left(\mathrm{T}_{\mathrm{F}}\right)$ that, in turn, is the primordial quantum field.

1.2. Pal et al [3] developed three different equations expressing the quantized energy ( $\boldsymbol{E}_{T}$ ) of TCP. The value of $\mathcal{E}_{T}$ in one of the three equations is shown below: 


$$
\varepsilon_{T}=4.384 \times 10^{-16} \mathrm{erg} \equiv 2.73 \times 10^{-4} \mathrm{eV} \equiv 2.73^{0} \mathrm{~K} \cong C M B R
$$

temperature $\equiv 2.725^{\circ} \mathrm{K}$

It is to be noted that $1 \mathrm{erg} \cong 0.6241807 \times 10^{12} \mathrm{eV}$ and $10^{-4} \mathrm{eV} \cong 1^{0} \mathrm{~K}$ (where $\mathrm{K}=$ Kelvin) as indicated by Weisskopf [6].

1.3. Pal et al [3] expressed that the CMBR temperature is due to $\varepsilon_{T}$, the quantized energy of TCP where $\varepsilon_{T}=4.384 \times 10^{-16} \mathrm{erg} \equiv 2.73^{\circ} \mathrm{K}$ that is very close to $2.725^{\circ} \mathrm{K}$ (CMBR temperature). As per Pal et al [3], it is interesting to note that all of the three different equations ultimately give rise to the same result that is equivalent to the CMBR temperature. A sort of relationship is thus observed between the $\varepsilon_{T}$ and the CMBR temperature. This coincidence is thus signifying a probable role of TCP on the maintenance of CMBR temperature. Further, this coincidence is also signifying the existence of these TCP in the presence of TRP. TCP cannot exist without TRP and vice versa. The presence of TCP, TRP and Thought force $\left(\mathrm{T}_{\mathrm{F}}\right)$ in the universe is thus indicated and expressed mathematically. At present we are unable to explain when and how these TCP and TRP decoupled from the primordial cosmic soup.

Pal et al $[1,3]$ showed that the quantized energy $\left(\varepsilon_{T}\right)$ of TCP is responsible to cause the universal consciousness as well as the cosmic microwave background radiation temperature. The individual consciousness owes its origin to the universal consciousness created by the same $\varepsilon_{T}$. Ultimately this $\mathcal{E}_{T}$ represents universal consciousness.

\subsection{General view for the evolution of the universe:}

Big Bang $\rightarrow$ Universe $\rightarrow$ [Fields + Particles \& Antiparticles + STC $] \rightarrow[$ Matter and Fields + STC $] \rightarrow$ Matter and Fields + STC + Life as well as consciousness.

If the universe exists along with the universal consciousness that, in turn, is a functional state of the universal mind (UM) and if this UM is evolved at the Big Bang from the eternal void, then

\section{Our view for the evolution of the universe (see Scheme-I)}

VOID $\rightarrow$ [Big Bang $] \rightarrow[$ TCP, TRP \& Anti-TRP in the inherent presence of thought force $\left.\left(\mathrm{T}_{\mathrm{F}}\right)+\mathrm{STC}\right] \rightarrow$ [Universal Mind with universal consciousness + TCP \& TRP in the inherent presence of thought force $\left.\left(\mathrm{T}_{\mathrm{F}}\right)+\mathrm{STC}+\mathrm{UTF}\right] \rightarrow$ [Universal Mind with universal consciousness + Matter and Fields \{including thought force $\left.\left(\mathrm{T}_{\mathrm{F}}\right)\right\}+\mathrm{TCP} \& \mathrm{TRP}+\mathrm{STC}+$ $\mathrm{UTF}] \rightarrow$ Universal Mind with universal consciousness + Matter and Fields \{including thought force $\left.\left(\mathrm{T}_{\mathrm{F}}\right)\right\}+$ Life as well as consciousness + TCP \& TRP + STC + UTF [where thought force $\left(\mathrm{T}_{\mathrm{F}}\right)$ is the origin of all the fields, TCP (Though-carrying particle) is the origin of all the field particles, TRP (Thought retaining particle) is the origin of all the matter particles, anti-TRP is the origin of all the anti-particles, STC $=$ Space time continuum and UTF $=$ Universal thought frequency $\cong$ frequency of the TCP].

\section{Thought Force}

Pal [5] explained that the quantized energy $\left(\varepsilon_{T}\right)$ of TCP is the energy responsible for generating thought force $\left(\mathrm{T}_{\mathrm{F}}\right)$; thus the $\mathrm{T}_{\mathrm{F}}$ may be expressed as

$$
\mathrm{TF}=\mathcal{E}_{T} / \mathrm{Di}
$$

where $\mathrm{D}_{\mathrm{i}}=$ Interacting distance.

The thought force $\left(\mathrm{T}_{\mathrm{F}}\right)$ has been postulated to be carried by TCP in the inherent presence of TRP. The TCP being the carrier of the $T_{F}$ would behave like bosons when TRP would function like fermions.

Depending on the $\mathrm{D}_{\mathrm{i}}$ (= Interacting distance), it is possible to calculate and identify two new forces viz. $\mathrm{T}_{\mathrm{F}}$ (micro) $[=$ Thought force in microcosm] and $\mathrm{T}_{\mathrm{F}}$ (macro) [= Thought force in macrocosm], the existence of which is indicated and expressed by Pal [5].

$\mathrm{Pal}$ [5] explained that Thought force $\left(\mathrm{T}_{\mathrm{F}}\right)$, an expression of the universal consciousness, is the primordial quantum field that, in turn, functions as the primary unified field. This $\mathrm{T}_{\mathrm{F}}$ being an expression of the universal consciousness is applicable to any inanimate object as well as to any biological system (having thinking ability). Thus the $\mathrm{T}_{\mathrm{F}}$ being an expression of the universal consciousness exerts its functions both in vitro and in vivo.

Physicists determined that underlying quantum fields give birth to elementary particles. Pal [5] expressed that the thought force $\left(\mathrm{T}_{\mathrm{F}}\right)$ is the primordial quantum field. Thought force $\left(T_{F}\right)$ being the primordial quantum field functions as the primary unified field. Thought force $\left(\mathrm{T}_{\mathrm{F}}\right)$ being the primordial quantum field gives birth to TRP that appears to be the origin of all the matter particles. TCP is the carrier of thought force $\left(\mathrm{T}_{\mathrm{F}}\right)$ that, in turn, appears to be the origin of all the fields. TCP thus appears to be the origin of all the field particles (see Scheme-I).

In a purpose to involve both the non-living and living systems of the world, Pal [5] has shown the existences of these TCP, TRP and thought force $\left(\mathrm{T}_{\mathrm{F}}\right)$ in vitro and thought force $\left(\mathrm{T}_{\mathrm{F}}\right)$ in vivo.

$\mathrm{Pal}$ (5) expressed that the non-living system of the world is governed by the thought force $\left(\mathrm{T}_{\mathrm{F}}\right)$ in vitro and this Thought force $\left(\mathrm{T}_{\mathrm{F}}\right)$ in vitro gives rise to $\mathrm{T}_{\mathrm{F}}$ (micro), $\mathrm{SNF}$, EMF, WNF, GF and $\mathrm{T}_{\mathrm{F}}$ (macro)

where $\mathrm{T}_{\mathrm{F}}($ micro $)=$ Thought force in microcosm, $\mathrm{SNF}=$ Strong nuclear force, $\mathrm{EMF}=$ Electromagnetic force, $\mathrm{WNF}=$ Weak nuclear force, $G F=$ Gravitational force and $T_{F}$ $($ macro $)=$ Thought force in macrocosm. It is to be noted here that $\mathrm{T}_{\mathrm{F}}$ (micro) is a stronger force than the $\mathrm{SNF}$ and $\mathrm{T}_{\mathrm{F}}$ (macro) is a weaker force even than the GF.

Pal (5) also expressed that the living system of the world is governed by the thought force $\left(\mathrm{T}_{\mathrm{F}}\right)$ in vivo and this Thought force $\left(\mathrm{T}_{\mathrm{F}}\right)$ in vivo is a type of force that represents the biological 'thought' which is the action of mind. This 'thought' being a type of force controls the 'thought processes' involving the firing of neurons through the quantum mechanical activities of these TCP and TRP in the 
presence of consciousness. This consciousness, in turn, is the quantized energy $\left(\varepsilon_{T}\right)$ of TCP. The thought force $\left(\mathrm{T}_{\mathrm{F}}\right)$ in vivo is demonstrated in numerous experiments in which thought has an effect on a physical process (often known as mind over matter). This biological 'thought' is a type of force that can cause movement. Controlling movement through thought alone is observed in several experiments conducted by many scientists as indicated by Pal (5). These experiments thus signify the existence of thought force $\left(\mathrm{T}_{\mathrm{F}}\right)$ in vivo.

Pal [5] explained the existence of $\mathrm{T}_{\mathrm{F}}$ (micro) $(=$ Thought force in microcosm). This $\mathrm{T}_{\mathrm{F}}$ (micro) is the strongest interaction (a new class of 'extra strong' interaction) indicated by Weinberg [7] and Quigg [8]. It is stronger than SNF (Strong Nuclear Force). And this $\mathrm{T}_{\mathrm{F}}$ (micro) confirms the existence of TCP in the inherent presence of TRP in the microcosm.

Pal [5] has also shown the existence of $\mathrm{T}_{\mathrm{F}}$ (macro) (= Thought force in macrocosm). It is the "weakest force" which is much weaker even than the gravity as explained by Perkins [9] and indicated by Weinberg [7]. This $\mathrm{T}_{\mathrm{F}}$ (macro) confirms the existence of TCP in the inherent presence of TRP into the quantum geometry of the universe.

\section{How to Define and Interpret 'Time'}

There is an arrow of time and there is something strange in the way time relates to consciousness. It is puzzling fact that the fundamental laws of both the quantum world as well as the large-scale universe are independent of time, and yet humans are actually aware of the arrow of time. Roger Penrose feels that this is a profound issue and this is the reason that both in classical physics and quantum physics there is no place for consciousness. In his book Shadows of the Mind Roger Penrose says, "Consciousness is a part of the universe". He believes that there is an objective world out there which exists whether it is perceived by a human mind or not, whose most remarkable manifestation, consciousness, is open to study by modern science. "Science will have to be quite seriously changed in order that consciousness can be accommodated". Penrose says, "We need something extended beyond that, where the idea of evolving time can actually make sense".

\section{As per Hawking [10], there are at least three different arrows of time}

(1) The cosmological arrow of time begins at the Big Bang, the beginning of the universe; because the earlier time before the Big Bang could not just be defined. This cosmological arrow of time is the direction of time in which the universe expands rather than contracts.

(2) The thermodynamic arrow of time is the direction of time in which the disorder or entropy increases.

(3) The psychological arrow of time is the direction of time in which we feel time passes: the direction in which we remember the past but not the future. The psychological arrow is essentially the same as the thermodynamic arrow because these two would always point in the same direction.

\subsection{What are the Characteristics of 'Time'}

Regarding the characteristics of "time", the following points are to be taken into account as indicated by Hawking [10]:

(1) The theory of relativity reveals that "time" is not absolute. It can expand or contract; nothing can travel faster than the speed of light.

(2) Time begins at the 'Big Bang' singularity at which the universe is supposed to begin.

(3) Time would come to an end at the 'Big Crunch' singularity at which the universe is supposed to come to an end.

(4) Time also comes to an end at the singularity inside a black hole, which is formed due to gravitational collapse of a star to infinite density.

(5) There is a scope to use anidea of "imaginary time" (by incorporating $i=\sqrt{-1}$ ) through which the distinction between time and space disappears completely.

(6) There is no singularity or boundary in imaginary time, leading to the conclusion: The boundary condition of the universe is that it has no boundary. In imaginary time, the time is indistinguishable from directions in space.

(7) If one can utilize the 'quantum theory of gravity' (by unifying gravity with quantum mechanics) along with the idea of "imaginary time", then one would be able to open up a new possibility, in which there would be no boundary to space-time and there would be no singularity at which all the laws of science usually break down. This will lead to the totally "self-contained" universe ........The universe is neither created nor destroyed.....it is eternal.

(8) The arrow of time: The psychological arrow $=$ The thermodynamic arrow which agrees well with the cosmological arrow of time.

(9) "Time" is not completely separate from and independent of "space", but is combined with "space" to form what is called "space-time continuum".

Although "time" is sometimes expressed in the dimension of "length" (such as "long time" or "short time"), yet the time may oscillate periodically, thereby, maintaining the character of a "wave" which appears to perpetuate eternally. And this oscillating periodic character of time can clarify the truth in the philosophically uttered popular statement: History repeats itself. This periodic or oscillating time involves change only within a cyclical pattern; so there is no sense of extension beyond that cycle. Every event in this observable universe occurs on the platform of time through which such an event is generally being expressed. The concept of personal time (psychological time) begins to function to a person at the onset of the function of his or her conscious mind. 


\section{Time and its Possible Relation with Classical Physics and Quantum Physics through the Developed Consciousness Model Involving TCP, TRP and Thought Force $\left(T_{F}\right)$}

In the theory of relativity there is no unique time, but instead each individual has his own personal measure of time that depends on where he is and how he is moving. Space and time are dynamic quantities and they not only affect but also are affected by everything that happens in the Universe. Hawking [10] mentioned, "Time is not completely separate from and independent of space but is combined with it to form what is called 'space-time continuum'.

According to the Einstein's theory of relativity, there is no unique "absolute time", but instead, each individual has his own personal measure of time that depends on where he is and how he is moving. It is therefore concluded that time can expand or contract as it is expressed by the world famous "Twins paradox" which runs like this: Keeping one of the twins on the earth, if the other fellow goes for a trip in a space-ship at nearly the speed of light, then, after returning from his space trip, he would see that he is much younger than the one who was staying on the earth. The explanation that is usually given for this expansion or contraction of time is that the time should run slower near a massive body like earth. This is due to the fact that when light travels upwards crossing earth's gravitational field, it looses energy and thus its frequency goes down, as the energy of light is directly proportional to its frequency. It would thus appear to someone high up that everything down below is consuming longer time to happen. Among the surprising effects of relativity are that a moving clock runs slower than a clock at rest, and that time in a mountain top runs faster than time at sea level, because gravity is stronger at sea level and gravity slows time down. This phenomenon leads to the "Twins paradox" if one has the idea of "absolute time" at the back of one's mind as it is mentioned by Hawking [10].

It can be shown that

$$
G_{F}=\frac{1}{K}\left(\varepsilon_{T} / R\right)^{2}=G / c^{4}\left(\varepsilon_{T} / R\right)^{2}=\left(G / c^{4}\right)\left[T_{F}(\text { macro })\right]^{2}(2)
$$

where $G_{F}=$ gravitational force,

$$
\mathrm{K}=c^{4} / G \text {, }
$$

$\mathrm{c}=$ free-space velocity of light,

$\mathrm{G}=$ Newton's gravitational constant,

$\mathcal{E}_{T}=$ quantized energy of the TCP radiated from the radiant mass of the universe,

$\mathrm{R}=$ radius of the universe $=1.482 \times 10^{29} \mathrm{~cm}$,

$c / H_{0}=\mathrm{L}$ (Hubble length) $=$ radius of the universe $=\mathrm{R}=$ $1.482 \times 10^{29} \mathrm{~cm}$,

$H_{0}=$ present value of Hubble's parameter =

\section{$2.023988 \times 10^{-19} \mathrm{sec}^{-1}$.}

$\mathrm{T}_{\mathrm{F}}($ macro $)=$ Thought force in macrocosm $=\boldsymbol{\varepsilon}_{T} / \mathrm{R}$.

The gravity may thus be related with $\mathcal{E}_{T}$, the quantized energy of TCP radiated from the radiant mass of the universe. Thus TCP and TRP once detected may shed immense light on the 'gravity' (even on 'black holes') in order to detect 'gravity waves', ripples in 'space-time' predicted by Einstein.

All the parameters on the right hand side of the Equation (2) are more or less physical constants. Even $\mathrm{T}_{\mathrm{F}}$ (macro) itself is a type of universal constant. Accepting $\mathrm{T}_{\mathrm{F}}$ (macro) to be a universal constant, the usual dimensional analysis of the Equation (2) would signify that

$$
G_{F} \propto \text { Time }^{4}
$$

The Equation (3) indicates that the "physical time" would have to expand with the increase in $\mathrm{G}_{\mathrm{F}}$ and similarly, the physical time would contract with the decrease in $\mathrm{G}_{\mathrm{F}}$.

The concept of 'psychological time' would thus have to expand to a person existing within the field of lower energy on the surface of the earth where there is a gravitational field, which has negative energy. Can this prediction of general relativity regarding the expansion of time near a massive body be explained by the fact that the time would have to expand to a person existing within a field of lower energy level relative to the other fellow who is existing (in the space-ship) within a field of higher energy level? This "contraction" and "expansion" of time indicate the presence of an inverse relationship between the "time" and "the intensity of the field of energy" where one is staying and how one is moving.

The nature of time's arrow has been discussed by physicists, philosophers, sages and charlatans but there is no agreement. The world of atomic particles shows us that there is really no time's arrow at the quantum level. "Time" itself loses its meaning, and at the Planck scale the words "before" and "after" are without application as indicated by Wheeler [11]. [Planck length $=\mathrm{L}=\left(h G / c^{3}\right)^{1 / 2}=4.04 \times 10^{-33} \mathrm{~cm}$ ].

Wheeler [11] pointed out, "Three-dimensional geometry carries information about time (Wheeler et al [12]). The geometry is not deterministic, even though it looks so at the every day scale of observation. Instead, at microscopic [or Planck length $\left.=\mathrm{L}=\left(h G / c^{3}\right)^{1 / 2}=4.04 \times 10^{-33} \mathrm{~cm}\right]$ scale, it "resonates" between one configuration and another and another. The ${ }^{(3)} G^{\prime} s$ with appreciable amplitude are too numerous to be accommodated into any one space-time. Thus the uncertainty principle declares that space-time is only an approximate and classical concept. In reality there is no such thing as space-time. "Time" itself loses its meaning, and [at the Planck scale] the words "before" and "after" are without application".

This is due to the fact that approximately $10^{15}$ times Planck length $\left(\approx 4.04 \times 10^{-33} \mathrm{~cm}\right)$ is required to arrive at the radius $\left(=8.28 \times 10^{-18} \mathrm{~cm}\right)$ of a TCP. It is to be noted that the theoretically calculated radius of a TCP or TRP 
within a nucleon $=8.28 \times 10^{-18} \mathrm{~cm}$. It has already been mentioned that the quantized energy $\left(\varepsilon_{T}\right)$ of TCP is responsible to cause the generation of consciousness. And further there is no manifestation of 'time' unless there is the generation of consciousness $=\varepsilon_{T}=m_{T} c^{2}=h c / \lambda_{T}$ (where $m_{T}=$ quantized mass of the TCP, $\mathrm{c}=$ free- space velocity of light, $\mathrm{h}=$ Planck's quantum constant, $\lambda_{T}=$ wavelength of the TCP).

The manifestation of any type of "time" is solely dependent on the manifestation of "consciousness" $\left(=\mathcal{E}_{T}\right)$. The concept of personal time (psychological time) begins to a person at the onset of the function of his or her "conscious mind" only. 'Time' is bound to the 'mind'. Even the existence of cosmological arrow of time is also dependent on the existence of universal consciousness that, in turn, is the quantized energy ( $\varepsilon_{T}$ ) of TCP. This $\mathcal{E}_{T}$ represents universal consciousness.

Consciousness is the functional state of mind. Presence of consciousness signifies the presence of mind and vice versa. It is to be understood that consciousness is the realization of existence and consciousness moves up and down on the 'scale of the realization' of existence through the 'sub-conscious', 'conscious' and 'super-conscious' states as indicated by Pal et al [2]. There is another term called unconscious state. The most usual unconscious state is sleep. A deeper form of unconsciousness is called a coma.

As 'time' is solely dependent on the manifestation of consciousness $\left(=\boldsymbol{E}_{T}\right)$, so time is absent to an unconscious person. Thus time is absent to a sleeping person. Similarly, time is absent to a person in coma state.

\section{TCP, TRP and Thought Force $\left(T_{F}\right)$ Play Significant Roles in the Evolution of Time}

The consciousness model of Pal et al [1-3] signifies that the universe exists along with the universal consciousness that is created by the quantized energy $\left(\varepsilon_{T}\right)$ of TCP. The thought force $\left(\mathrm{T}_{\mathrm{F}}\right)$ is an expression of the universal consciousness.

The quantized energy $\left(\varepsilon_{T}\right)$ of TCP is responsible to cause the universal consciousness as well as the cosmic microwave background radiation temperature. Pal et al [3] expressed that the presence of CMBR temperature indicates the existence of TCP in the presence of TRP. The existence of TCP being the carrier of thought force $\left(\mathrm{T}_{\mathrm{F}}\right)$ ensures the existence of thought force $\left(T_{F}\right)$. The thought force $\left(T_{F}\right)$ being an expression of the universal consciousness is liable to function as the primordial quantum field. Further, this $\mathrm{T}_{\mathrm{F}}$ being an expression of the quantized energy ( $\boldsymbol{\varepsilon}_{T}$ ) of TCP exerts its functions both in vitro and in vivo.

These TCP, TRP and thought force $\left(\mathrm{T}_{\mathrm{F}}\right)$ in vitro and $\left(\mathrm{T}_{\mathrm{F}}\right)$ in vivo play their significant roles both in the non-living and living systems of this universe, although it is hard at present to prove their physical existence thoroughly except the maintenance of CMBR temperature and the existence of thought force $\left(\mathrm{T}_{\mathrm{F}}\right)$ in vivo. The controlling movement through thought alone is observed in several experiments conducted by Birbaumer [13], Donoghue et al [14-17], Hatsopoulos [18] and Miguel [19]. These experiments thus signify the positive proof for existence of the TCP, TRP and thought force $\left(\mathrm{T}_{\mathrm{F}}\right)$ in vivo. The existence of the thought force ( $\mathrm{T}_{\mathrm{F}}$ ) in vivo confirms the presence of $\boldsymbol{\varepsilon}_{T}$. This $\boldsymbol{\varepsilon}_{T}$ is the quantized energy of TCP radiated by the radiant mass of this universe.

Pal et al [3] indicated that these TCP and TRP are the ultimate constituents of any matter as well as any mind in the inherent presence of the thought force $\left(\mathrm{T}_{\mathrm{F}}\right)$ in vitro and thought force $\left(\mathrm{T}_{\mathrm{F}}\right)$ in vivo. Consciousness is defined here as the 'self-organized' capability of any living being to activate TCP and TRP, the ultimate constituents of mind and matter.

Pal et al $[1,3]$ explained that the eternal 'Void' has been covered by the mind to function as the Soul of individual

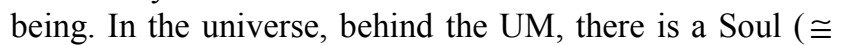
VOID). In the individual, behind the individual mind (which

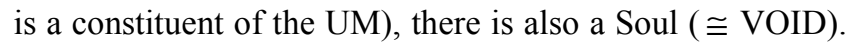

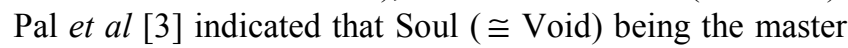
ruler of the body exerts its functions through the finer instrument called mind. The mind (with the help of TCP and TRP) exerts its functions through the brain and spinal cord (CNS) along with the other parts of the nervous system (PNS along with ANS) in the biological systems in order to grasp this physical universe, of course, in the inherent presence of consciousness. This consciousness itself is acting as an inter-linking agent between the animate and inanimate through the quantum mechanical activities of these TCP and TRP. Thus these TCP and TRP being the ultimate constituents of mind as well as matter play the most significant roles in many present day scientific enigmas. The universal consciousness is expressed here as the quantized energy $\left(\varepsilon_{T}\right)$ of TCP radiated by the radiant mass of this universe. The individual consciousness owes its origin to the universal consciousness created by the same $\varepsilon_{T}$.

As per Edelman, a Nobel Laureate, consciousness is a process, a chain of events, rather than a 'thing' contained in a special part of the brain. Pal et al $[1,3]$ expressed that consciousness is a process involving the quantum mechanical activities of these TCP and TRP that are the ultimate constituents of any matter as well as any individual mind in the inherent presence of thought force $\left(\mathrm{T}_{\mathrm{F}}\right)$ in vitro and thought force $\left(\mathrm{T}_{\mathrm{F}}\right)$ in vivo.

It is our plausible opinion that the manifestation of time is simply and solely dependent on the generation of consciousness that is the quantized energy $\left(\boldsymbol{\varepsilon}_{T}\right)$ of TCP. This $\varepsilon_{T}$ represents universal consciousness.

Lynds [20] says, "It should become clear that no matter how small the time interval, or how slowly an object moves during that interval, it is still in motion and its position is constantly changing, so it can't have a determined relative position at any time. Indeed, if it did, it couldn't be in motion. 
Thus all the motion paradoxes stand as mere mathematical tricks and can be explained in my theory. It is something entirely subjective that we project onto the world around us. That is, it is the outcome of brain function and consciousness".

In our proposition, the manifestation of time is absolutely dependent on the generation of consciousness (= $\left.\mathcal{E}_{T}\right)$. That is why Lynds [20] has to point out that time is the outcome of brain function and consciousness [that is the quantized energy $\left(\mathcal{E}_{T}\right)$ of TCP].

Georgiev [21] expressed, "Observed rare cases in the clinical practice of patients with time agnosia clearly show that consciousness is possible without perception of time and that for these patients apparent dynamics of observed objects do not produce feeling of "time flow", as intuitively expected. Thus if our mind operates at $100 \mathrm{GHz}$ (dynamical timescale of 10 picoseconds), every single "occasion of consciousness" experienced as "present" could be result from $10^{10}-10^{11}$ conscious steps sharing the same time label". He further pointed out, "Recently proposed models of boson condensation as mind process (with $10^{-14} \mathrm{~S}$ dynamics) cannot provide any biological function taking part at this timescale that is why I think the $100 \mathrm{GHz}$ conscious informational processing is the fastest possible".

\section{Discussion and Conclusion}

\subsection{Discussion}

As per Georgiev [21], our mind operates at $100 \mathrm{GHz}$ (dynamical timescale of 10 picoseconds) where $100 \mathrm{GHz}=$ $10^{11} \mathrm{~Hz}$ and 10 picoseconds $=10^{-11}$ sec. It can be shown that the calculated frequency of TCP $=0.6612 \times 10^{11} \mathrm{~Hz}$ that agrees well with $100 \mathrm{GHz}\left(=10^{11} \mathrm{~Hz}\right)$. And the calculated time period of the TCP $=1.51 \times 10^{-11} \mathrm{sec}$ that is equivalent to 10 picoseconds $=10^{-11} \mathrm{sec}$. It is thus apparent that there is a remarkable relationship between the operational mind and the quantum mechanical activities of these TCP and TRP with which every mind is assumed to be constituted. Thus our assumption that mind is constituted and operated by these TCP and TRP is found to be correct through these propositions and experimental observations.

At the onset of the Big Bang, the time as well as space is generated along with the universal mind (UM) with the universal consciousness (see Scheme-I). Thus, at the onset of the Big Bang, the time is generated along with the space which itself is gradually taking its own usual characteristic 'shape' to accommodate subsequently the formation of matter within itself by the projection and activity of TCP and TRP in the inherent presence of thought force $\left(\mathrm{T}_{\mathrm{F}}\right)$. This thought force $\left(\mathrm{T}_{\mathrm{F}}\right)$ is an expression of the universal consciousness that, in turn, is the quantized energy ( $\mathcal{E}_{T}$ ) of TCP.

The TCP in the inherent presence of TRP plays the fundamental role for the initiation of consciousness ( $\varepsilon_{T}=m_{T} c^{2}=h c / \lambda_{T}$ ) through which the evolution of light (at the Big Bang) and its velocity (c) can be realized. Thus, the apparent origin of the physical time appears to be the origin of evolution of light and its evolved velocity (c) at that point of the beginning of the universe.

Light travels fast and far, but even the light stops at a black hole. A black hole is a star that is so massive that it collapses to a practically no volume under its own gravity. The gravitational force in a black hole is so intense that even light cannot escape from it. It is to be noted that time (as well as space) becomes zero at the inside singularity of a black hole. The origin of the physical time thus appears to be related with the origin of the evolution of light and its evolved velocity (c). Thus, the velocity (c) of light as well as time is always related with consciousness = $\varepsilon_{T}=m_{T} c^{2}=h c / \lambda_{T}$. And the consciousness is thus conjectured here to be the 'mental light'. It is to be noted that $\mathcal{E}_{T}$ is found to be directly proportional to $\mathrm{c}$ (free-space velocity of light) where the proportionality constant, $K=h / \lambda_{T}$ (where h is

Planck's quantum constant $=6.63 \times 10^{-27} \mathrm{erg} . \mathrm{sec}$, and $\lambda_{T}$ is the wavelength in $\mathrm{cm}$ of TCP).

Pal et al [2] explained, "Consciousness is the realization of existence, and there are as many states of consciousness as there are states of existence. Every living being has a consciousness of its own depending on complexity of the brain and the activity of the viable numbers of TCP in the presence of TRP; and the state of its consciousness changes every moment of time. Consciousness is the perception of the relation it bears to things and as this relation changes, consciousness changes its character. Consciousness itself does not change; it only moves up and down on the 'scale of the realization' of existence through the 'sub-conscious', 'conscious' and 'super-conscious' states".

\subsection{Conclusion}

Time of any kind cannot exist without the existence of consciousness. Time without consciousness is not possible. But consciousness without time is possible. That is why Georgiev [21] pointed out, "Consciousness is possible without perception of time." It is really very difficult to understand the fact that consciousness alone can exist independently without perception of time. Consciousness can exist independently in the form of super-consciousness, a state that is usually enjoyed and experienced by a real 'YOGI' (who practises meditation). In the super-conscious state, the perception of time becomes zero. It also indirectly signifies the existence of consciousness prior to space, time, and substance. It thus indicates the existence of the universal consciousness that, in turn, exists along with the universe, thereby, proving the fact that consciousness, not matter, is the ground of all existence.

Consciousness, not matter, is the ground of all existence, declares University of Oregon physicist Goswami through his published (1993) book, "The Self-Aware Universe: How Consciousness Creates the Material World". He accepts the Vedantic view and holds that the universe is self-aware, and that consciousness creates the physical world. Matter is an 
expression of mind, not separate from mind, but mind manifested materially.

It is evident that when we remain vigorously absorbed in some kind of deep thought or when we remain in the exulted ecstatic mood (thereby existing within the high energy level mentally and internally) being vigorously involved with the beloved persons, then we feel that the time runs very quickly. In other words, the personal concept of time is contracted as the intensity of the field of energy is increased. On the other hand, the time appears to run very slowly (that is the time is expanded) when we fall in the distressed and hopeless condition in which we remain within the exhausted energy level mentally and internally. Thus whenever we remain in the state of high concentration of mind, thereby existing within a high energy level mentally and internally, we usually forget the existence of time. Time is contracted ultimately to zero. We then forget the existence of time altogether, although we are not really conscious about the intrinsic meaning of this vanishing of time and re-appearing of time.

\section{References}

[1] D. Pal and A.U. De, Physics of consciousness and its model may provide guidelines to solve many scientific problems. Neuroquantology 1, 17-28 (2004)

[2] D. Pal, and A.U. De, Consciousness model: Significance of thought-carrying particles and thought-retaining particles in quantum measurement as well as cognitive problem. NeuroQuantology 2, 115-116 (2005)

[3] D. Pal, and A.U. De, The cosmic microwave background radiation temperature signifying the existence of the thought-carrying particle, thought retaining particle and thought force. NeuroQuantology 10: Issue3; 428-442 (September 2012)

[4] M. Bhaumik, Code Name GOD. (Penguin Books India Pvt. Ltd., 11 Community Centre, Panchsheel Park, New Delhi 110 017, India) pp. 132-133; 161-162; 167; 171; 177; 185$186 ; 89-190 ; 198$ (2006)

[5] D. Pal, Existence of thought force and its characteristics. Communicated to American Journal of Modern Physics (AJMP) (2013)

[6] V. F. Weisskopf, The Origin of the Universe, The World of Physics, (Simon and Schuster, 1230 Avenue of Americas, New York 10020) 3, pp. 10 (1987)

[7] S. Weinberg, Conceptual Foundations of the Unified Theory of Weak and Electromagnetic Interactions. The World of Physics, (Simon and Schuster, 1230 Avenue of Americas, New York 10020); 3, pp.164 and 165 (1987)
[8] C. Quigg, Elementary Particles and Forces. The World of Physics, (Simon and Schuster, 1230 Avenue of Americas, New York 10020); 2, $884-891$ (1987)

[9] D. H. Perkins, Introduction to High Energy Physics. (Addition-Wesley Publishing Co., Massachusetts 01867, U.S.A.) pp. 17 and 364 (1982)

[10] S. W. Hawking, A Brief History of Time from the Big Bang to Black Holes. (Bantam Books, 666 fifth Avenue, New York 10103) pp. 15-36; 141-152 and 153-160 (1989)

[11] J. A. Wheeler, "Time today", Proceedings of workshop, "Physical origin of Time Asymmetry". (Cambridge University Press) (1993).

[12] R. Baierlain, H. Sharp, and J. Wheeler, Three-dimensional geometry as carrier of information about time. Phys. Rev. 126, pp. 1864 to 1865 (1962)

[13] N. Birbaumer, N. Ghanayim, T. Hinterberger, I. Iversen, B. Kotchoubey, A. Kübler, J. Perelmouter, E. Taub, and H. Flor, A spelling device for the paralysed. Nature 398, Number 6725; 297-9825 (March 1999)

[14] J. P. Donoghue, L. R. Hochberg, M. D. Serruya, G. M. Friehs, J. A. Mukand, M. Saleh, A. H. Caplan, A. Branner, D. Chen, and R. D. Penn, Neuronal ensemble control of Prosthetic Devices by a Human with tetraplegia. Nature 422, 13; 164-171 (July 2000)

[15] J. P. Donoghue, Connecting cortex to machines: recent advances in brain Interfaces, Nature Neuroscience Supplement. 5 1085-8 (November 2002)

[16] J. P. Donoghue, D. Mijail, G. N. Serruya, L. P. Hatsopoulos, F. R. Matthew, Brain-machine interface: Instant neural control of a movement signal. Nature 416, 14 Number 6877 , 141 (March 2002)

[17] J. P. Donoghue, R. K. Aaron, H. M. Herr, D. McK Ciombor, L. R. Hochberg, C. L. Briant, J. R. Morgan, and M. G. Ehrlich, Horizons in prosthesis development for restoration of Limb Function. Am Accad Ortho Surg September. 14 (10) S198-S204 (2006)

[18] M. G. Hatsopoulos, D. Rubino, and K. A. Robbins, Propagating waves Mediate information transfer in the motor cortex. Nature Neuroscience. 9 1549-1557 (2006)

[19] Nicolelis MAL Miguel, Brain-Machine interfaces to restore motor function and probe neural circuits. Nature Reviews Neuroscience. 4 417-422 (2003)

[20] P. Lynds, Time and Classical and Quantum Mechanics: Indeterminacy Versus Discontinuity. Foundations of Physics Letters. 16, Number- 4, 343-355 (2003)

[21] D. D. Georgiev, Consciousness operates beyond the timescale for discerning time intervals: implications for Q-mind theories and analysis of quantum decoherence in brain. NeuroQuantology 2, pp. 136 and 140-141 (2004) 